\title{
Novel Techniques to Reduce Search Space in Multiple Minimum Supports-Based Frequent Pattern Mining Algorithms
}

\author{
R. Uday Kiran \\ International Institute of Information \\ Technology-Hyderabad \\ Hyderabad \\ Andhra Pradesh, India \\ uday_rage@research.iiit.ac.in
}

\author{
P. Krishna Reddy \\ International Institute of Information \\ Technology-Hyderabad \\ Hyderabad \\ Andhra Pradesh, India \\ pkreddy@iiit.ac.in
}

\begin{abstract}
Frequent patterns are an important class of regularities that exist in a transaction database. Certain frequent patterns with low minimum support (minsup) value can provide useful information in many real-world applications. However, extraction of these frequent patterns with single minsupbased frequent pattern mining algorithms such as Apriori and FP-growth leads to "rare item problem." That is, at high minsup value, the frequent patterns with low minsup are missed, and at low minsup value, the number of frequent patterns explodes. In the literature, "multiple minsups framework" was proposed to discover frequent patterns. Furthermore, frequent pattern mining techniques such as Multiple Support Apriori and Conditional Frequent Pattern-growth (CFP-growth) algorithms have been proposed. As the frequent patterns mined with this framework do not satisfy downward closure property, the algorithms follow different types of pruning techniques to reduce the search space. In this paper, we propose an efficient CFP-growth algorithm by proposing new pruning techniques. Experimental results show that the proposed pruning techniques are effective.
\end{abstract}

\section{Categories and Subject Descriptors}

H.2.8 [Database Management]: Database Applications Data Mining

\section{General Terms}

Algorithms

\section{Keywords}

Data mining, knowledge discovery, frequent patterns and multiple minimum supports.

Permission to make digital or hard copies of all or part of this work for personal or classroom use is granted without fee provided that copies are not made or distributed for profit or commercial advantage and that copies bear this notice and the full citation on the first page. To copy otherwise, to republish, to post on servers or to redistribute to lists, requires prior specific permission and/or a fee.

EDBT 2011, March 22-24, 2011, Uppsala, Sweden

Copyright 2011 ACM 978-1-4503-0528-0/11/0003 ...\$10.00

\section{INTRODUCTION}

Since the introduction of frequent patterns in [1], the problem of mining frequent patterns from the transaction databases has been actively studied in the literature [4]. Most of the frequent pattern mining algorithms (e.g., Apriori [2] and FP-growth [5]) use "single minimum support (minsup) framework" to discover complete set of frequent patterns. Minsup controls the minimum number of transactions a pattern must cover in a database. The frequent patterns discovered with this framework satisfy downward closure property. That is, "all non-empty subsets of a frequent pattern must also be frequent." This property holds the key for minimizing the search space in all of the single minsup-based frequent pattern mining algorithms [2, 4].

Most of the real-world databases are non-uniform in nature containing both frequent and rare items. A rare item is an item having low frequency. Frequent patterns containing rare items can provide useful information to the users.

Example 1: In a supermarket, costly goods such as Bed and Pillow are less frequently purchased than the cheaper goods such as Bread and Jam. However, the association between the former set of items can be more interesting as it may generate relatively more revenue.

However, mining frequent patterns containing both frequent and rare items with "single minsup framework" leads to the rare item problem which is as follows: At high minsup, the frequent patterns containing rare items will be missed, and at low minsup, combinatorial explosion can occur, producing too many frequent patterns.

To confront rare item problem, an effort has been made in [10] to find frequent patterns with "multiple minsups framework." In this framework, each pattern can satisfy a different minsup depending upon the items within it. The frequent patterns discovered through "multiple minsups framework" do not satisfy downward closure property. As a result, this property cannot be used for minimizing the search space in multiple minsups-based frequent pattern mining algorithms.

In the literature, an Apriori-like algorithm known as Multiple Support Apriori (MSApriori) was proposed to find frequent patterns with "multiple minsups framework" [10]. Also, an FP-growth-like algorithm known as Conditional Frequent Pattern-growth (CFP-growth) has been proposed to mine frequent patterns [6]. Since downward closure property no 
longer holds in "multiple minsups framework," the CFPgrowth algorithm has to carry out exhaustive search in the constructed Tree structure. In this paper we propose an improved CFP-growth algorithm, called CFP-growth++, by introducing four pruning techniques to reduce the search space. Experimental results on various types of datasets show that the proposed algorithm is efficient and scalable as well.

\subsection{Related Work}

The occurrence of rare item problem with the usage of traditional data mining techniques to discover knowledge involving rare items was introduced in [13]. In [10], "multiple minsups framework" has been introduced to address rare item problem, and MSApriori algorithm was proposed for extracting frequent patterns. An FP-growth-like algorithm [5], called CFP-growth [6], has been proposed to mine frequent patterns. It was shown that the performance of CFP-growth is better than the MSApriori algorithm. In [14], a new interestingness measure, called relative support has been introduced, and an Apriori-like algorithm has been proposed for mining frequent patterns containing both frequent and rare items. An Apriori-like approach which tries to use a different minsup at each level of iteration has been discussed in [11]. A stochastic mixture model based on negative binomial distribution has been discussed to mine rare association rules [3]. An approach has been suggested to mine the association rules by considering only infrequent items i.e., items having support less than the minsup [16].

We have been investigating improved approaches to mine frequent patterns containing both frequent and rare items. In [8], an improved methodology has been proposed to specify items' $M I S$ values. In [9], a new interestingness measure, called item-to-pattern difference, has been used along with the "multiple minsups framework" to discover frequent patterns in the databases, where frequencies of the items' vary widely. An effort has been made to extend the notion of multiple constraints to extract periodic-frequent patterns [12]. In [7], we have proposed a preliminary algorithm to improve the performance of CFP-growth by suggesting two pruning techniques for reducing the size of constructed tree structure. It is to be noted that the algorithm discussed in [7] performs exhaustive search, like CFP-growth, to discover complete set of frequent patterns as the frequent patterns mined with "multiple minsups framework" do not satisfy downward closure property.

In this paper, we investigated approaches to reduce the search space while extracting frequent patterns and proposed two additional pruning techniques which significantly reduces the search space by avoiding exhaustive search while extracting frequent patterns from a tree structure. Overall, we have proposed a comprehensive algorithm by employing four pruning techniques to efficiently mine frequent patterns.

\subsection{Paper Organization}

The remaining part of the paper is organized as follows. In Section 2, we explain the necessary background. In Section 3, we discuss the CFP-growth algorithm and its performance issues. In Section 4, we discuss the proposed pruning techniques to reduce the search space and present the CFPgrowth ++ algorithm. Experimental results are discussed in Section 5. The last section contains conclusions and future work.

\section{BACKGROUND}

In this section, we explain the basic model of frequent patterns, rare item problem and the extended model of frequent patterns based on multiple minsups.

\subsection{Basic Model of Frequent Patterns}

Frequent patterns were first introduced in [1]. The basic model of frequent patterns is as follows:

Let $I=\left\{i_{1}, i_{2}, \cdots, i_{n}\right\}$ be a set of items, and a transaction database $D B=\left\langle T_{1}, T_{2}, \cdots, T_{n}\right\rangle$, where $T_{i}(i \in[1 . . n])$ is a transaction which contains a set of items in $I$. Each transaction is associated with an identifier, called TID. The support of a pattern (or an itemset) $X$, denoted as $S(X)$, is the number transactions containing $X$ in $D B$. The pattern $X$ can be frequent if its support is no less than a userdefined minimum support (minsup) threshold value, i.e., $S(X) \geq$ minsup. A pattern containing $k$ number of items is a $k$-pattern. The support of a pattern can also be represented in percentage of $|D B|$. In this paper, we use the terms "itemset" and "pattern" interchangeably.

Example 2: Consider the transaction database of 20 transactions shown in Table 1 . The set of items $I=$ $\{a, b, c, d, e, f, g, h\}$. The set of $a$ and $b$, i.e., $\{a, b\}$ is a pattern. It is a 2-pattern. For simplicity, we write this pattern as ' $a b$ '. It occurs in tids of $1,4,7,10,11,13,16$ and 19. Therefore, the support of $a b, S(a b)=8$. If the user-specified minsup $=6$, then $a b$ is a frequent pattern because $S(a b) \geq$ minsup.

Table 1: A transaction database.

\begin{tabular}{|l|l|}
\hline TID & Items \\
\hline 1 & $\mathrm{a}, \mathrm{b}$ \\
\hline 2 & $\mathrm{a}, \mathrm{e}, \mathrm{f}$ \\
\hline 3 & $\mathrm{c}, \mathrm{d}$ \\
\hline 4 & $\mathrm{a}, \mathrm{b}, \mathrm{h}$ \\
\hline 5 & $\mathrm{c}, \mathrm{d}$ \\
\hline 6 & $\mathrm{a}, \mathrm{c}$ \\
\hline 7 & $\mathrm{a}, \mathrm{b}$ \\
\hline 8 & $\mathrm{e}, \mathrm{f}$ \\
\hline 9 & $\mathrm{c}, \mathrm{d}, \mathrm{g}$ \\
\hline 10 & $\mathrm{a}, \mathrm{b}$ \\
\hline 12 & $\mathrm{a}, \mathrm{b}$ \\
\hline 13 & $\mathrm{a}, \mathrm{c}$ \\
\hline 14 & $\mathrm{~b}, \mathrm{e}, \mathrm{f}, \mathrm{g}$ \\
\hline 15 & $\mathrm{c}, \mathrm{d}$ \\
\hline 16 & $\mathrm{a}, \mathrm{b}, \mathrm{d}$ \\
\hline 17 & $\mathrm{c}, \mathrm{d}$ \\
\hline 18 & $\mathrm{a}, \mathrm{c}$ \\
\hline 20 & $\mathrm{a}, \mathrm{b}, \mathrm{e}$ \\
\hline
\end{tabular}

Apriori [1] and FP-growth [5] are the two popular algorithms to mine frequent patterns. Apriori uses candidategenerate-and-test-approach to discover the complete set of frequent patterns. FP-growth employs pattern-growth technique to discover complete set of frequent patterns. In the literature, it has been shown that FP-growth performs better than Apriori [5].

\subsection{Rare Item Problem}

Real-world databases are mostly non-uniform in nature containing both frequent and relatively infrequent (or rare) items. If the items' frequencies in a database vary widely, we encounter the following issues while mining frequent patterns under single minsup framework:

i. If minsup is set too high, we will miss the frequent patterns containing rare items.

ii. To find frequent patterns that involve both frequent and rare items, we have to set low minsup. However, 
this may cause combinatorial explosion, producing too many frequent patterns, because those frequent items will combine with one another in all possible ways and many of them are meaningless.

This dilemma is called the rare item problem.

Example 3: Consider the database shown in Table 1. At high minsup, say minsup $=6$, we will miss the frequent patterns involving the rare items ' $e$ ' and ' $f$ '. To mine the frequent patterns containing ' $e$ ' and ' $f$ ', we have to specify low minsup value. Let the minsup value be 3 . The frequent patterns discovered at minsup $=3$ are shown in the fourth column of the Table 2. Among the generated frequent patterns, the pattern ' $a c$ ' can be considered uninteresting to the user because it has low support and contains frequently occurring items ' $a$ ' and ' $c$ '. This pattern can be considered interesting if it has satisfied high minsup, say minsup $=6$.

Table 2: Frequent patterns generated at minsup $=3$. The terms "S", "MIS", "SMF" and "MMF" are respectively used as the acronyms to denote support, minimum item support, "single minsup framework" and "multiple minsups framework." The terms "T" and "F" respectively denote the frequent patterns generated and have not generated in single and multiple minsups frameworks.

\begin{tabular}{|l|l|l|c|c|}
\hline Patterns & $S$ & $M I S$ & SMF & MMF \\
\hline$a$ & 12 & 10 & $\mathrm{~T}$ & $\mathrm{~T}$ \\
\hline$c$ & 9 & 10 & $\mathrm{~T}$ & $\mathrm{~F}$ \\
\hline$b$ & 9 & 8 & $\mathrm{~T}$ & $\mathrm{~T}$ \\
\hline$d$ & 7 & 6 & $\mathrm{~T}$ & $\mathrm{~T}$ \\
\hline$e$ & 4 & 3 & $\mathrm{~T}$ & $\mathrm{~T}$ \\
\hline$f$ & 3 & 3 & $\mathrm{~T}$ & $\mathrm{~T}$ \\
\hline$a b$ & 8 & - & $\mathrm{T}$ & $\mathrm{T}$ \\
\hline$a c$ & 3 & - & $\mathrm{T}$ & $\mathrm{F}$ \\
\hline$c d$ & 6 & - & $\mathrm{T}$ & $\mathrm{T}$ \\
\hline$e f$ & 3 & - & $\mathrm{T}$ & $\mathrm{T}$ \\
\hline
\end{tabular}

\subsection{Extended Model of Frequent Patterns}

To confront the rare item problem, an effort has been made in the literature to extend the basic model of frequent patterns to multiple minsups [10]. In the extended model, each item in the transaction database is specified with a support constraint known as minimum item support $(M I S)$ and minsup of a pattern is represented with the minimal $M I S$ value among all its items (see Equation 1).

$$
\operatorname{minsup}(X)=\operatorname{minimum}\left(\begin{array}{cc}
M I S\left(i_{1}\right), & M I S\left(i_{2}\right), \\
\cdots, & M I S\left(i_{k}\right)
\end{array}\right)
$$

where, $X=\left\{i_{1}, i_{2}, \cdots, i_{k}\right\}, 1 \leq k \leq n$, is a pattern and $M I S\left(i_{j}\right), 1 \leq j \leq k$, represents the $M I S$ of an item $i_{j} \in X$.

The extended model enables the user to simultaneously specify high minsup for a pattern containing only frequent items and low minsup for a pattern containing rare items. Thus, efficiently addressing the rare item problem. The significance of this model is illustrated in Example 4.
Example 4: Continuing with Example 3, let the userspecified $M I S$ values for the items ' $a$ ', ' $b$ ', ' $c$ ', ' $d$ ', ' $e$ ', ' $f$ ', ' $g$ ' and ' $h$ ' be $10,8,10,6,3,3,3$ and 2 , respectively. The items' $M I S$ values are specified with respect to their support values. The frequent patterns discovered with the extended model are shown in the fifth column of Table 2. It can be observed that the uninteresting frequent pattern ' $a c$ ' that was generated at low minsup (i.e., at minsup $=3$ ) in Example 3 has failed to be a frequent pattern in this model. It is because $S(a c)<$ minsup (= minimum $(M I S(a), \operatorname{MIS}(c))$.

\section{CFP-GROWTH AND PERFORMANCE IS- SUES}

In [10], an Apriori-like algorithm known as Multiple Support Apriori (MSApriori) has been discussed to mine frequent patterns. An FP-growth-like algorithm known as Conditional Frequent Pattern-growth (CFP-growth) has been discussed to efficiently mine frequent patterns [6]. Among the two algorithms, it has been shown that CFP-growth algorithm performs better than MSApriori algorithm. In this section we discuss CFP-growth and its performance issues.

\subsection{CFP-growth}

The CFP-growth algorithm is developed based on the FPgrowth algorithm [6]. Even though it is an FP-growth-like algorithm, the structure, construction and mining procedures of CFP-growth are different from FP-growth. The CFP-growth algorithm accepts transaction database and $M I S$ values of items as an input. Using the items' $M I S$ values as prior knowledge, it discovers complete set of frequent patterns with a single scan on the transaction database. Briefly, the working of CFP-growth is as follows.

i. Items are sorted in descending order of their MIS values. Using the sorted list of items, an FP-tree-like structure known as MIS-tree is constructed with a single scan on the transaction database. Simultaneously, the support of each item in the MIS-tree is measured.

ii. To reduce the search space, tree-pruning operation is performed to prune the items that cannot generate any frequent pattern. The criterion used is prune the items that have support less than the lowest $M I S$ value among all items.

Table 3: MIS and support values of items.

\begin{tabular}{|l|l|l|l|l|l|l|l|l|}
\hline Items & $a$ & $b$ & $c$ & $d$ & $e$ & $f$ & $g$ & $h$ \\
\hline MIS & 10 & 8 & 10 & 6 & 3 & 3 & 3 & 2 \\
\hline Support & 12 & 9 & 9 & 7 & 4 & 3 & 2 & 1 \\
\hline
\end{tabular}

Example 5: Table 3 provides information about the $M I S$ and support values of items present in the database of Table 1 . The lowest MIS value among all items is 2 . Therefore, it is clear that no pattern will have minsup less than 2. Based on apriori property [2], it turns out that ' $h$ ' and its supersets cannot generate any frequent pattern as their supports will be no more than 1. So, CFPgrowth prunes ' $h$ ' from the MIS-tree. 
iii. After tree-pruning operation, tree-merging operation is performed on the MIS-tree to merge the child nodes of a parent node that share same item. The resultant MIS-tree is called compact MIS-tree.

iv. Finally, choosing each item in the compact MIS-tree as the suffix item (or pattern), its conditional pattern base (i.e., prefix sub-paths) is build to discover complete set of frequent patterns. Since frequent patterns do not satisfy downward closure property, CFP-growth tries to discover complete set of frequent patterns by building suffix patterns until its respective conditional pattern base is empty.

Example 6: Consider the compact MIS-tree shown in Figure 2(b). For the (suffix) item ' $f$ ', the conditional prefix paths are $\langle a, e: 1\rangle,\langle e: 1\rangle$ and $\langle b, e: 1\rangle$. The CFP-growth algorithm builds the suffix patterns $\langle f\rangle,\langle f, e\rangle,\langle f, b\rangle,\langle f, a\rangle,\langle f, e, b\rangle$ and $\langle f, e, a\rangle$ to discover the complete set of frequent patterns.

\subsection{Performance Issues}

The performance issues of CFP-growth algorithm are as follows.

First, the criterion used by CFP-growth to construct compact MIS-tree still considers some items which cannot generate any frequent pattern at higher-order.

Example 7: Continuing with Example 5, CFP-growth constructs compact MIS-tree with the items ' $a$ ', ' $b$ ', ' c', ' $d$ ', ' $e$ ', ' $f$ ' and ' $g$ '. However, item ' $g$ ' cannot generate any frequent pattern at higher-order because its support (i.e., 2) is less than the lowest $M I S$ value (i.e., 3) among all the items ' $a$ ', ' $b$ ', 'c', ' $d$ ', ' $e$ ', ' $f$ ' and ' $g$ '.

Second, as CFP-growth continues to build suffix patterns until its respective conditional pattern base is empty, CFPgrowth searches in some of those (infrequent) suffix patterns which will never generate any higher-order frequent pattern.

Example 8: Continuing with Example 6, the lowest $M I S$ value among the items ' $a$ ', ' $b$ ', ' $e$ ' and ' $f$ ' is $3(=M I S(f))$. Since the support of ' $a$ ' and ' $b$ ' in the conditional pattern base of ' $f$ ' is less than 3 , it is straight forward to prove that $\{f, a\}$ and $\{f, b\}$ cannot be frequent patterns. In addition, their supersets also cannot be frequent patterns. Thus, CFP-growth spends additional resources (i.e., runtime) to discover the complete set of frequent patterns.

\section{PROPOSED APPROACH}

In this section, we first introduce the properties and theorems that have been identified for reducing the search space. Next, we explain the pruning techniques to reduce the search space and present the algorithm.

\subsection{Theorems}

The pruning techniques that are proposed for reducing the search space in the "multiple minsups framework" are based on apriori property (see Property 1) and Theorems 4.1 and 4.2

Property 1. (Apriori property.) In a database DB, if $X$ and $Y$ are two patterns such that $X \subseteq Y$, then $S(X) \geq$ $S(Y)$.
THEOREM 4.1. In every frequent pattern, the item having lowest MIS value is a frequent item.

Proof. Consider a transaction database $D B$ containing the set of items, $I=\left\{i_{1}, i_{2}, \cdots, i_{n}\right\}$, such that $M I S\left(i_{1}\right) \geq$ $M I S\left(i_{2}\right) \geq \cdots \geq \operatorname{MIS}\left(i_{n}\right)$. Let $X=\left\{i_{j}, \cdots, i_{k}\right\} \subseteq \bar{I}$, where $1 \leq j \leq k \leq n$, be a pattern. If $X$ is frequent, then $S(X) \geq \operatorname{minimum}\left(M I S\left(i_{j}\right), \cdots, M I S\left(i_{k}\right)\right)$. That is, $S(X) \geq M I S\left(i_{k}\right)$. From Property 1 , it turns out that $S\left(i_{k}\right) \geq S(X) \geq M I S\left(i_{k}\right)$. Thus, if $X$ is frequent, then $i_{k}$ is a frequent item.

THEOREM 4.2. In every frequent pattern, all non-empty subsets containing the item having lowest MIS value will be frequent.

Proof. Consider a transaction database $D B$ containing the set of items, $I=\left\{i_{1}, i_{2}, \cdots, i_{n}\right\}$. Let $M I S\left(i_{j}\right)$, where $i_{j} \in I$, be the user-specified $M I S$ values such that $M I S\left(i_{1}\right) \geq$ $M I S\left(i_{2}\right) \geq \cdots \geq \operatorname{MIS}\left(i_{n}\right)$. Let $X=\left\{i_{j}, \cdots, i_{k}\right\} \subseteq I$, where $1 \leq j \leq k \leq n$, be a frequent pattern. That is, $S(X) \geq \operatorname{MIS}\left(i_{k}\right)\left(=\right.$ minimum $\left.\left(M I S\left(i_{j}\right), \cdots, M I S\left(i_{k}\right)\right)\right)$. Let $A \subset X$ be a pattern such that $i_{k} \in A$. Since $i_{k}$ has the lowest $M I S$ value among all items in $X$, it turns out that $\operatorname{minsup}(A)=M I S\left(i_{k}\right)$. From Property 1 , it can be derived that $S(A) \geq S(X) \geq M I S\left(i_{k}\right)$. Thus, $A$ is a frequent pattern.

\subsection{Techniques to Reduce the Search Space}

We propose four techniques to reduce the search space.

\subsubsection{Least minimum support}

In the multiple minsups framework, each pattern can satisfy a different minsup depending upon the items within it. The term least minimum support $(L M S)$ refers to the lowest minsup of all frequent patterns. Since frequent item is a frequent 1-pattern, it is straight forward to prove from Theorem 4.1 that $L M S$ is always equal to the lowest $M I S$ value among all frequent items. $L M S$ has the following two properties.

Property 2. If $X=\left\{i_{1}, i_{2}, \cdots, i_{k}\right\} \subseteq I$, where $1 \leq$ $k \leq n$, is a pattern such that $S(X)<L M S$, then $S(X)<$ minimum $\left(M I S\left(i_{1}\right), M I S\left(i_{2}\right), \cdots, M I S\left(i_{k}\right)\right)$.

Property 3. If $X$ and $Y$ are two patterns such that $X \subset$ $Y$ and $S(X)<L M S$, then $S(Y)<L M S$.

These two properties facilitate to use $L M S$ as a constraint to reduce the search space. In particular, $L M S$ can be used to prune the items (or patterns) that cannot generate any frequent pattern at higher-order. The significance of $L M S$ is illustrated in Example 9.

Example 9: Continuing with Example 5, the frequent items in the transaction database of Table 1 are ' $a$ ', ' $b$ ', ' $d$ ', ' $e$ ' and ' $f$ '. Based on Theorem 4.1, it can be said that any frequent pattern that is mined from this database will have one of the above items as the item having lowest $M I S$ value. Thus, lowest minsup that can be satisfied by a frequent pattern is lowest $M I S$ value among all these frequent items i.e., 3 . Since, the items ' $g$ ' and ' $h$ ' have support less than 3 , their supersets also cannot have support greater than 3 (Property $1)$. Thus, it is guaranteed that ' $g$ ' and ' $h$ ' cannot generate any frequent pattern at higher-order. 


\subsubsection{Conditional Minimum Support}

Let Tree be the FP-tree-like structure constructed after scanning a database in $M I S$ descending order of items. If we consider an item $i_{j}$ that exists in Tree as a suffix item (or 1pattern) and construct its prefix sub-paths (i.e., conditional pattern base), then $M I S$ of $i_{j}$ will be the lowest $M I S$ value among all the items in the conditional pattern base. From the definition of minsup in multiple minsups framework, it turns out that any frequent pattern that is going to be generated from the conditional pattern base of $i_{j}$ should satisfy MIS value of $i_{j}$. Thus, we call the MIS value of the suffix item $i_{j}$ as the conditional minsup. The correctness of this idea is shown in Lemma 4.3.

Lemma 4.3. Let $\alpha$ be a pattern in MIS-tree and $S_{\alpha}$ be the

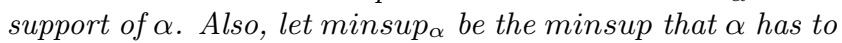
satisfy, $B$ be $\alpha$ 's conditional pattern base, and $\beta$ be an item in $B$. The support of $\beta$ in $B$ be $S_{B}(\beta)$ and $M I S_{\beta}$ be the $\beta$ 's MIS value. The minsup of pattern $\langle\alpha, \beta\rangle$ is minsup m

Proof. According to the definition of MIS-tree, $M I S_{B}(\beta)$ will always be greater than or equal to the minsup $_{\alpha}$. Therefore, minsup of $\langle\alpha, \beta\rangle$ is minsup $\alpha$.

Example 10: Consider the compact MIS-tree shown in Figure 2(b). For the (suffix) item $f$, the conditional prefix paths are $\langle\mathrm{a}$, e: 1$\rangle,\langle\mathrm{e}: 1\rangle$ and $\langle\mathrm{b}$, e: $1\rangle$. The item having lowest $M I S$ value among all the items ' $a$ ', ' $b$ ', ' $e$ ' and ' $f$ ' is ' $f$ ' which is the suffix item. As a result, every frequent pattern that gets generated from the conditional pattern base of ' $f$ ' will have minsup $=M I S(f)$. Thus, $M I S(f)$ is considered as conditional minsup for mining frequent patterns from the conditional pattern base of the suffix item ' $f$ '.

\subsubsection{Conditional Closure Property}

Property 4. (Conditional Closure property.) If a suffix pattern is infrequent, then all its super-suffix patterns (i.e., suffix pattern along with other item(s) in its conditional pattern base) will also be infrequent.

The correctness of this property is shown in Lemma 4.4.

Lemma 4.4. Let $\alpha$ be a pattern in MIS-tree and $S_{\alpha}$ be the

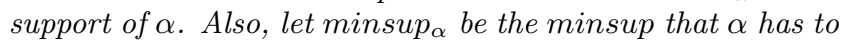
satisfy, $B$ be $\alpha$ 's conditional pattern base, and $\beta$ be an item in $B$. The support of $\beta$ in $B$ be $S_{B}(\beta)$ and $M I S_{\beta}$ be the $\beta$ 's MIS value. If $\alpha$ is infrequent, then the pattern $\langle\alpha, \beta\rangle$ is also infrequent.

Proof. According to the definition of conditional pattern base and MIS-tree, each subset in $B$ occurs under the condition of the occurrence of $\alpha$ in the transaction database. If an item $\beta$ appears in $B$ for $n$ times, it appears with $\alpha$ in $n$ times. From the definition of frequent pattern used in the minimum constraint model, the minsup of $\langle\alpha, \beta\rangle$ is minimum $\left(\operatorname{minsup}_{\alpha}, M I S_{\beta}\right)=$ minsup $_{\alpha}$. As $S_{\alpha}<$ minsup $_{\alpha}$, the $S_{\langle\alpha, \beta\rangle}<\operatorname{minsup}_{\alpha}$ (apriori property [1]). Therefore, $\langle\alpha, \beta\rangle$ is also infrequent.

\subsubsection{Infrequent leaf node pruning}

The leaf nodes of a Tree that belong to infrequent items can be pruned without missing any frequent pattern or changing the support of a frequent pattern. We call this pruning technique as "infrequent leaf node pruning." It is straight forward to prove from the "conditional minsup" and conditional closure property that the conditional pattern base of a suffix item that is infrequent will not result in any frequent pattern.

\subsection{CFP-growth++}

The proposed CFP-growth++ algorithm is an improvement over CFP-growth algorithm. It successfully addresses the above two issues of CFP-growth. The differences between CFP-growth and CFP-growth++ are as follows:

i. The CFP-growth++ employs a better criterion to identify the items that cannot generate any frequent pattern. This criterion enables CFP-growth++ to construct compact MIS-tree with only those items that can generate frequent patterns.

ii. The proposed algorithm will not search for frequent patterns until the conditional pattern base of a suffix pattern is empty. Instead, it tries to identify which suffix patterns can generate frequent patterns at higher order and perform search only in them.

The CFP-growth++ algorithm accepts transaction database $D B$, set of items $I$ and items' $M I S$ values as the input parameters. Using the items' $M I S$ values as the prior knowledge, CFP-growth ++ discovers the complete set of frequent patterns with a single scan on the transaction database. The steps involved in CFP-growth++ are as follows: (i) construction of MIS-tree (ii) generating compact MIS-tree and (iii) mining frequent patterns from the compact MIS-tree.

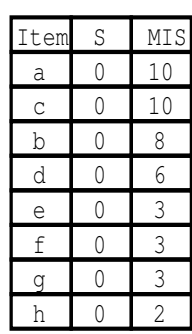

(a)

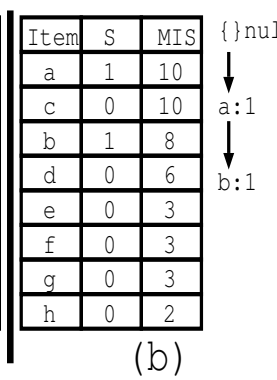

(b)
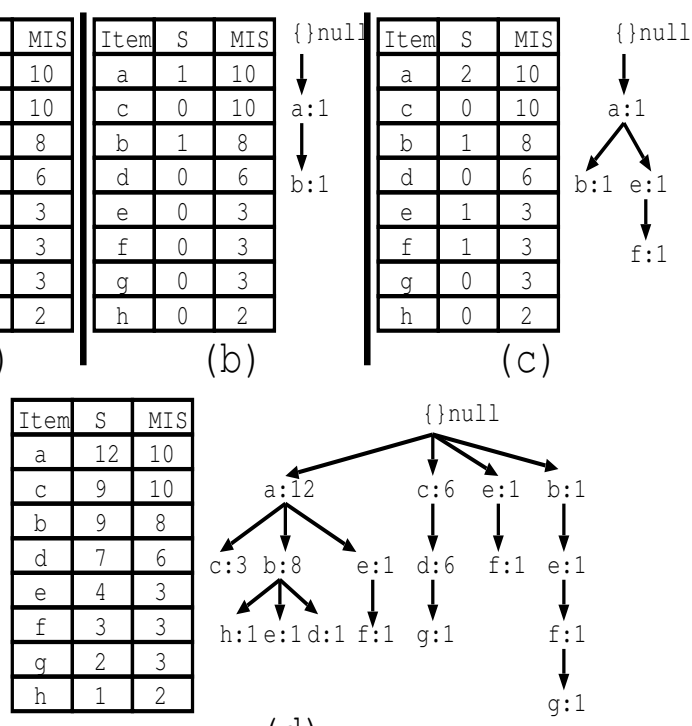

(d)

Figure 1: Construction of MIS-tree. (a) Initial MISlist (b) After scanning first transaction (c) After scanning second transaction and (d) After scanning every transaction.

\subsubsection{Construction of MIS-tree}

The $M I S$-tree consists of two components: $M I S$-list and prefix-tree. The MIS-list is a list having three fields - 
$\overline{\text { Algorithm } 1 \text { MIS-tree ( } D B \text { : transaction database, } I \text { : item- }}$ set containing $n$ items, MIS: minimum item support values for $n$ items)

1: Let $L$ represent the set of items sorted in decreasing order of their MIS values.

2: In $L$ order, insert items into the MIS-list with $S=0$ and mis equivalent to the respective $M I S$ value.

3: Create the root of a MIS-tree, $T$, and label it as "null".

4: for each transaction $t \in D B$ do

5: $\quad$ Sort all the items in $t$ in $L$ order.

6: Count the support values of any item $i$, denoted as $S(i)$ in $t$.

7: Let the sorted items in $t$ be $[p \mid P]$, where $p$ is the first element and $P$ is the remaining list. Call InsertTree $([p \mid P], T)$.

\section{8: end for}

9: Let $j=n-1$.

10: for $(; j \geq 0 ; j=j-1)$ do

11: if $\left(S\left[\overline{i_{j}}\right]<M I S\left[i_{j}\right]\right)$ then

12: $\quad$ Delete the item $i_{j}$ in header table.

13: $\quad$ Call MisPruning $\left(\right.$ Tree,$\left.i_{j}\right)$.

14: else

15: $\quad L M S=M I S\left[i_{j}\right]$.

16: break.

17: end if

18: end for

19: for $(; j \geq 0 ; j=j-1)$ do

20: $\quad$ if $\left(S\left[i_{j}\right]<L M S\right)$ then

21: $\quad$ Delete the item $i_{j}$ in header table.

22: $\quad$ Call MisPruning $\left(\right.$ Tree,$\left.i_{j}\right)$.

23: $\quad$ end if

24: end for

25: Name the resulting table as MinFrequentItemHeaderTable.

26: Call MisMerge(Tree).

27: Call InfrequentLeaf NodePruning(Tree).

item name (item), support $(S)$ and minimum item support $(M I S)$. The structure of the prefix-tree in $M I S$-tree is same as that in FP-tree [5]. However, the difference is that items in the prefix-tree of FP-tree are arranged in descending order of their support values, whereas items in the prefix-tree of $M I S$-tree are arranged in descending order of their $M I S$ values. To facilitate tree-traversal, node-links are maintained in the $M I S$-tree as in FP-tree.

The construction of MIS-tree in CFP-growth++ algorithm is shown in Algorithm 1. We illustrate this algorithm by using the transaction database shown in Table 1 . Let the user-specified $M I S$ values for the items ' $a$ ', ' $b$ ', ' $c$ ', ' $d$ ', ' $e$ ', ' $f$ ', ' $g$ ' and ' $h$ ' be $10,8,10,7,3,3,3$ and 2 , respectively.

The items are sorted in descending order of their MIS values. Let this sorted list of items be $L$. Thus, $L=$ $\{a, c, b, d, e, f, g, h\}$ (Step 1 of Algorithm 1). In $L$ order, insert each item into the $M I S$-list with support equal to zero and $M I S$ equivalent to their respective $M I S$ value (Step 2 of Algorithm 1). The resultant MIS-list is shown in Figure $1(\mathrm{a})$.

A $M I S$-tree is then created as follows. First, a root node is created with label "null." The database $D B$ is scanned. The items in each transaction are processed in $L$ order, and a branch is created for each transaction as in FP-growth [5]. Simultaneously, we increment the support values of the re-
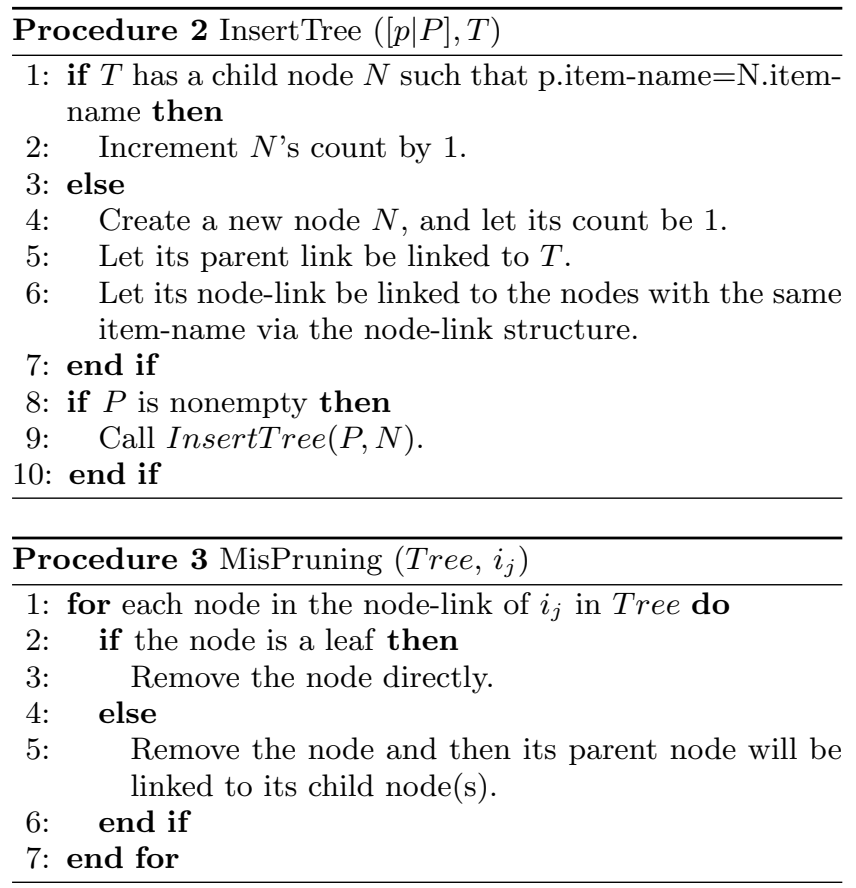

spective items in the MIS-list by 1 (Lines 4 to 8 in Algorithm 1 and Procedure 2). For example, the scan of the first transaction, " 1 : a,b" which contains two items $\langle\mathrm{a}, \mathrm{b}$ in $L$ order $\rangle$, leads to the construction of the first branch of the tree with two nodes $\langle\mathrm{a}: 1\rangle$ and $\langle\mathrm{b}: 1\rangle$, where ' $a$ ' is linked as a child of the root and ' $b$ ' is linked as the child node of ' $a$ '. Next, we increment support values of ' $a$ ' and ' $b$ ' in the MIS-list by 1 . The MIS-tree generated after scanning the first transaction is shown in Figure 1(b). The second transaction containing the items ' $a$ ', ' $e$ ' and ' $f$ ' in $L$ order will result in a branch where ' $a$ ' is linked to root, ' $e$ ' is linked to ' $a$ ' and ' $f$ ' is linked to ' $e$ '. However, this branch would share a common prefix, ' $a$ ', with the existing path for 1 . Therefore, we instead increment the count of ' $a$ ' node by 1 , and create new nodes, $\langle\mathrm{e}: 1\rangle$ and $\langle\mathrm{f}$ : 1$\rangle$, where ' $e$ ' is linked to ' $a$ ' and ' $f$ ' is linked to ' $e$ '. In the MIS-list, the support of the items ' $a$ ', ' $e$ ' and ' $f$ ' are incremented by 1 . The resultant MIS-tree is shown in Figure 1(c). Similar process is repeated for the remaining transactions and MIS-tree is updated accordingly. The resultant MIS-tree after scanning every transaction in the transaction database is shown in Figure 1(d). For the simplicity of figures, we do not show the node traversal pointers in trees, however, they are maintained as in the construction process of FP-tree.

\subsubsection{Construction of compact MIS-tree}

The compact MIS-tree is generated by pruning those items from the MIS-tree that cannot generate any frequent pattern. The pruning techniques, $L M S$ and infrequent leaf node pruning, are used in this process. The procedure used for constructing compact MIS-tree is as follows.

The MIS-tree is constructed with every item in the transaction database. To decrease the search space, we use $L M S$ as a constraint to prune the items that cannot generate any frequent pattern. A method to prune such items from the MIS-tree is as follows.

i. Starting from the last item of the MIS-list, the items 

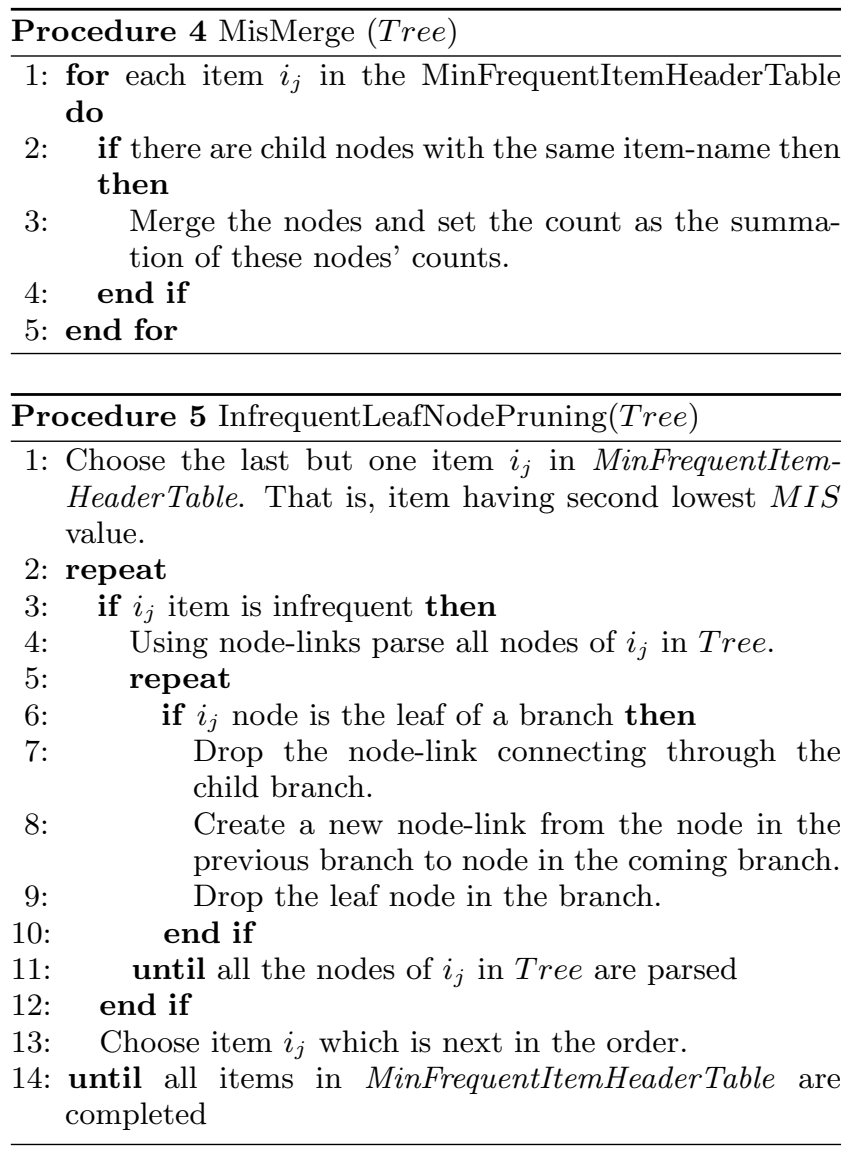

that have support less than their respective $M I S$ value are pruned (Lines 9 to 13 in Algorithm 1 and Procedure 3).

ii. Once the frequent item is found, its $M I S$ value is chosen as the $L M S$ value (Lines 14 to 18 in Algorithm $1)$. Next, support of the remaining items in the MISlist are compared with $L M S$ value, and those items that have support less than $L M S$ are pruned from the MIS-tree (Lines 19 to 24 in Algorithm 1 and Procedure $3)$.

We explain the construction of compact MIS-tree by considering the MIS-tree shown in Figure 1(d). The process starts from ' $h$ ' as it is the last item in the MIS-list of MIStree. This item is an infrequent item, therefore, it is pruned from the MIS-tree. Among the remaining items in the MISlist, ' $g$ ' is the last item in the MIS-list. It is also an infrequent item, therefore, it is pruned from the MIS-tree. Now, the last item in the MIS-list is ' $f$ '. It is a frequent item. Hence, no tree-pruning operation is carried for the item ' $f$.' Next, using the $M I S$ of the item ' $f$ ' as the $L M S$ value, the supports of the remaining items in the MIS-tree are compared. As these items have support greater than or equal to $L M S$ value, the tree-pruning operation ends.

After tree-pruning, tree-merging process is carried out to merge the child nodes of a parent node that share a common item (line 26 in Algorithm 1 and Procedure 4). The resultant MIS-tree is called compact MIS-tree. The compact MIS-tree generated after tree-pruning and tree-merging operations is shown in Figure 2(a). The process of infrequent leaf node
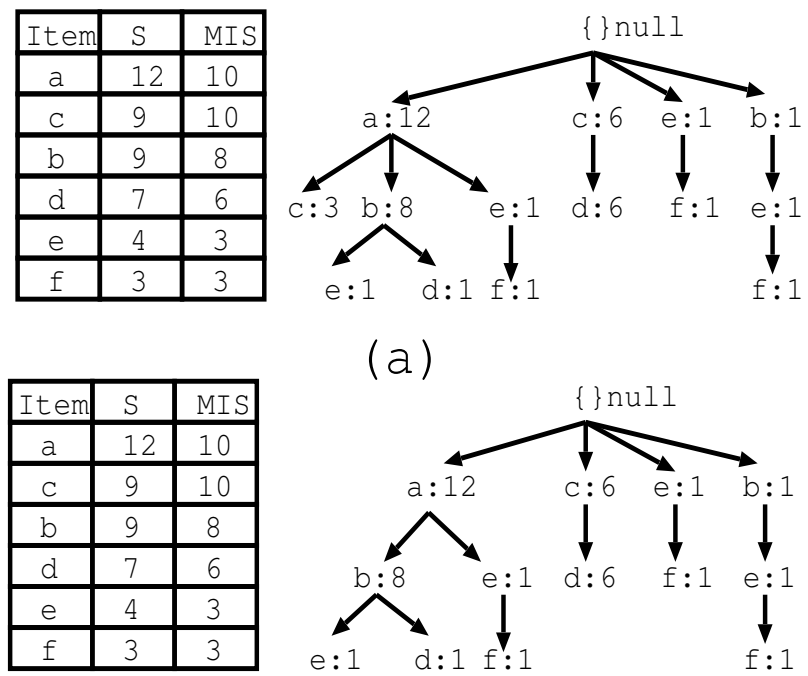

(a)

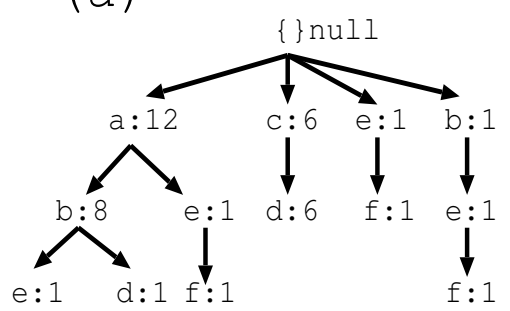

(b)

Figure 2: Compact MIS-tree. (a)After pruning items $g$ and $h$ and (b) After infrequent item leaf node pruning.

pruning (line 27 in Algorithm 1 and Procedure 5) is carried on the compact MIS-tree to decrease its size. The process is as follows. Among the remaining items in the MIS-list of MIS-tree, ' $c$ ' is an infrequent item (i.e., its support is less than the required minsup value). Therefore, using the node-links of ' $c$ ', we collect all the branches containing ' $c$ '. The branches containing $c$ are $\{\{\mathrm{a}, \mathrm{c}: 3\},\{\mathrm{c}, \mathrm{d}: 6\}\}$. In the branch $\langle\mathrm{a}, \mathrm{c}: 3\rangle, c$ is the leaf node, therefore, we prune the node ' $c$ ' in this branch. The resultant MIS-tree is shown in Figure 2(b). Note that the node of ' $c$ ' in the branch $\{\{c, d$ : $6\}\}$ it not pruned as it is not a leaf node. (Pruning ' $c$ ' in this branch will result in missing the frequent pattern ' $c d$ ' because $S(c d) \geq$ minimum $(M I S(c), M I S(d))$.

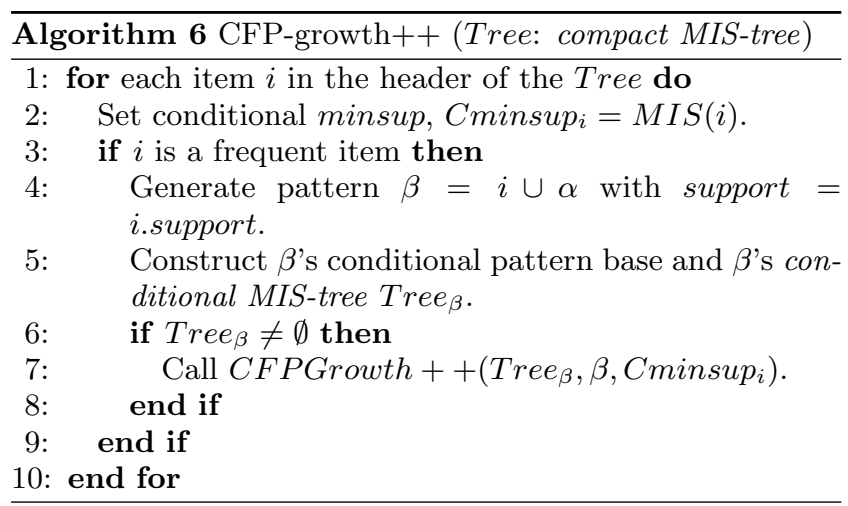

\subsubsection{Mining frequent patterns from compact MIS-tree}

The procedure for mining frequent patterns from compact MIS-tree is shown in Algorithm 6. The pruning techniques conditional minsup and conditional closure property are for mining frequent patterns.

The process of mining frequent patterns from the compact MIS-tree of Figure 2(b) is shown in Table 4 and is described 


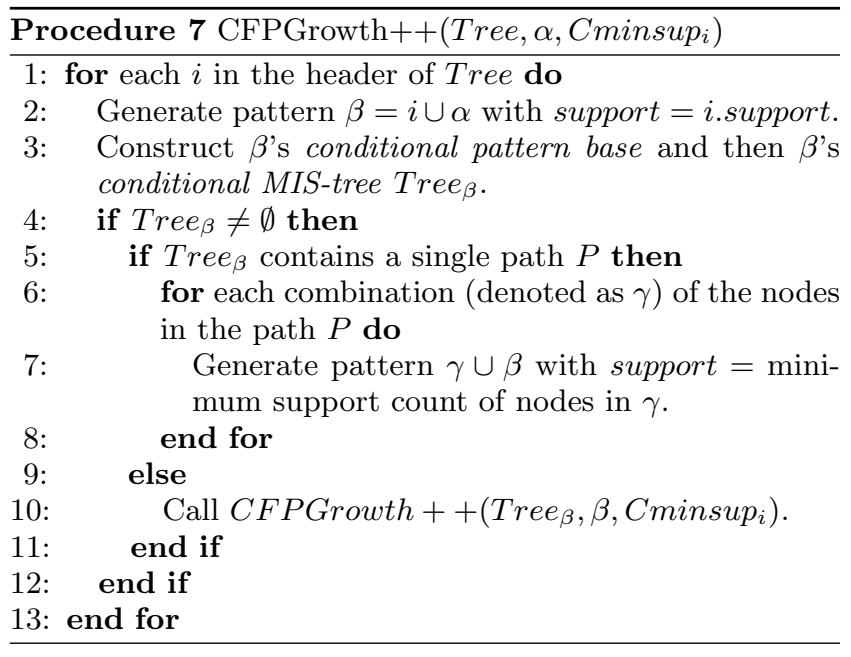

as follows. Consider the item ' $f$ ' that has lowest $M I S$ among all items in the compact MIS-tree. It occurs in 3 branches of compact MIS-tree. The branches are $\langle\mathrm{a}, \mathrm{e}, \mathrm{f}: 1\rangle,\langle\mathrm{e}, \mathrm{f}$ : $1\rangle$ and $\langle\mathrm{b}, \mathrm{e}, \mathrm{f}: 1\rangle$. Considering ' $f$ ' as a suffix pattern (or item), its conditional prefix paths are $\langle\mathrm{a}, \mathrm{e}: 1\rangle,\langle\mathrm{e}: 1\rangle$ and $\langle\mathrm{b}$, e: 1$\rangle$, which form its conditional pattern base. As the compact MIS-tree is constructed in MIS descending order of items, ' $f$ ' (suffix item) will have lowest $M I S$ value among all the items in its conditional pattern base. Therefore, using MIS value of the item ' $f$ ' (i.e., 3) as the conditional minsup, conditional MIS-tree is generated with $\langle\mathrm{e}: 3\rangle$; the items $a$ and $b$ are not included because the support counts are less than the specified conditional minsup value (i.e., conditional closure property). The single path generates the frequent pattern $\{\mathrm{e}, \mathrm{f}: 3\}$. Similar process is repeated for other remaining items in the compact MIS-tree to discover the complete set of frequent patterns.

Table 4: Mining compact MIS-tree by using multiple minsups and conditional pattern bases. The terms 'SI', ' $M S$ ' and 'Cond.' respectively denote 'suffix item', 'conditional minsup' and 'Conditional'.

\begin{tabular}{|l|l|l|l|l|}
\hline SI & $M S$ & $\begin{array}{l}\text { Cond. } \\
\text { pattern bases }\end{array}$ & $\begin{array}{l}\text { Cond. } \\
\text { MIS-tree }\end{array}$ & $\begin{array}{l}\text { frequent } \\
\text { patterns }\end{array}$ \\
\hline $\mathrm{f}$ & 3 & $\begin{array}{l}\langle\mathrm{a}, \mathrm{e}: 1\rangle\langle\mathrm{e}: 1\rangle \\
\langle\mathrm{b}, \mathrm{e}: 1\rangle\end{array}$ & $\langle\mathrm{e}: 3\rangle$ & $\{\{\mathrm{e}, \mathrm{f}: 3\}\}$ \\
\hline $\mathrm{e}$ & 3 & $\begin{array}{l}\langle\mathrm{a}, \mathrm{b}: 1\rangle\langle\mathrm{a}: 1\rangle \\
\langle\mathrm{b}: 1\rangle\end{array}$ & - & - \\
\hline $\mathrm{d}$ & 6 & $\langle\mathrm{a}, \mathrm{b}: 1\rangle\langle\mathrm{c}: 6\rangle$ & $\langle\mathrm{c}: 6\rangle$ & $\{\{\mathrm{c}, \mathrm{d}: 6\}\}$ \\
\hline $\mathrm{b}$ & 8 & $\langle\mathrm{a}: 8\rangle$ & $\langle\mathrm{a}: 8\rangle$ & $\{\{\mathrm{a}, \mathrm{b}: 8\}\}$ \\
\hline $\mathrm{c}$ & 10 & - & - & - \\
\hline
\end{tabular}

\section{EXPERIMENTAL RESULTS}

In this section, we evaluate the performance of FP-growth, CFP-growth and CFP-growth++ algorithms. We are not considering Apriori and MSApriori algorithms for comparison because it has been shown that FP-growth and CFPgrowth algorithms are better than the corresponding Apriori and MSApriori algorithms, respectively $[5,6]$.

The algorithms are written in GNU $\mathrm{C}++$ and run with Ubuntu 10.04 operating system on a $2.66 \mathrm{GHz}$ machine with
1GB memory. The experiments are pursued on synthetic $\left(T 10 I_{4} D 100 K\right)$ and real-world datasets (BMS-WebView-1 [15], Mushroom and Kosarak). T10I4D100K, BMS-WebView1 and Kosarak are sparse datasets and Mushroom is a dense dataset. These datasets are widely used in the literature for evaluating the performance of data mining algorithms. The datasets are available at Frequent Itemset Mining repository (http://fimi.cs.helsinki.fi/data/). The details of the datasets are shown in Table 5 .

Table 5: Dataset characteristics. The terms "max," "avg," and "trans" respectively denote maximum, average and transactions.

\begin{tabular}{|l|l|l|l|l|}
\hline Dataset & $\begin{array}{l}\text { Transa- } \\
\text { ctions }\end{array}$ & $\begin{array}{l}\text { Distinct } \\
\text { Items }\end{array}$ & $\begin{array}{l}\text { Max. } \\
\text { Trans. } \\
\text { Size }\end{array}$ & $\begin{array}{l}\text { Avg. } \\
\text { Trans. } \\
\text { Size }\end{array}$ \\
\hline T10I4D100k & 100000 & 870 & 29 & 10.102 \\
\hline BMS-WebView-1 & 59602 & 497 & 267 & 2.5 \\
\hline Mushroom & 8124 & 119 & 23 & 23 \\
\hline Kosarak & 990002 & 41270 & 2498 & 8.1 \\
\hline
\end{tabular}

In the experiment, we used the methodology discussed in [10] to assign items' $M I S$ values. The methodology is as follows:

$$
\operatorname{MIS}\left(i_{j}\right)=\operatorname{maximum}\left(\beta \times f\left(i_{j}\right), L S\right)
$$

The $f\left(i_{j}\right)$ and $M I S\left(i_{j}\right)$ variables respectively denote the frequency (or support) and minimum item support for an item $i_{j} \in I$. The variable $L S$ represents the user-specified least minimum item support allowed. In this, $\beta \in[0,1]$ is a parameter that controls how the MIS values for items should be related to their frequencies. If $\beta=0$, we have only one minimum support, $L S$, which is the same as the minsup in traditional frequent pattern mining. If $\beta=1$ and $f\left(i_{j}\right) \geq L S$, then $M I S\left(i_{j}\right)=f\left(i_{j}\right)$.

\subsection{Experiment 1}

In this experiment, both $L S$ and minsup values are set at $0.1 \%$ for T10I $4 D 100 K$ and BMS-WebView-1 datasets. For the Mushroom dataset, both $L S$ and minsup values are set at $10 \%$ as it is a dense dataset. To show how $\beta$ affects the number of frequent patterns found and the performance of the algorithms, we fixed $\beta=\frac{1}{\alpha}$ and varied $\alpha$. In the sparse datasets $(T 10 I 4 D 100 k$ and $B M S$-WebView- 1$), \alpha$ is varied from 1 to 20 . In the dense dataset (Mushroom), $\alpha$ is varied from 1 to 5 .

The experimental results regarding how the number of frequent patterns vary with the $M I S$ values in different datasets are shown in the Figures 3(a), 3(b) and 3(c). When $\alpha$ becomes larger, the number of frequent patterns found by the method gets closer to the number of frequent patterns found with the single minsup framework. The reason is as follows. At higher values of $\alpha$, the items' $M I S$ values become equals to $L S$. As a result, the performance of the "multiple minsups framework" is same as the "single minsup framework" with minsup $=L S$. It can also be observed that the above phenomenon happens at higher values of $\alpha$ in the sparse datasets (Figure 3(a) and 3(b)) and at lower values of $\alpha$ in the dense dataset (Figure 3(c)).

The experimental results about the runtime performance of FP-growth, CFP-growth and CFP-growth++ algorithms 


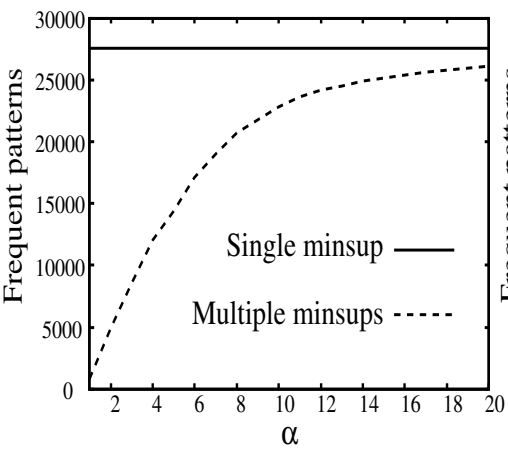

(a) T1014D100K dataset

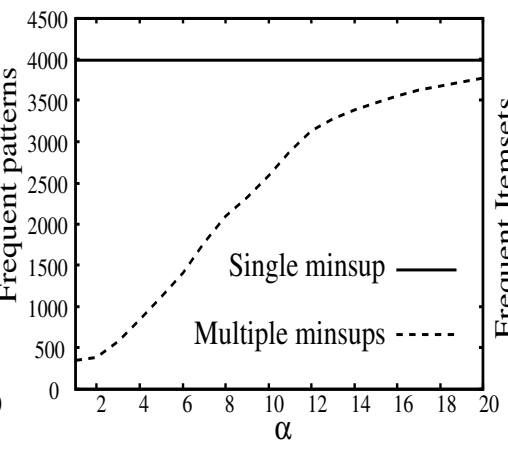

(b) BMS-WebView-1 dataset

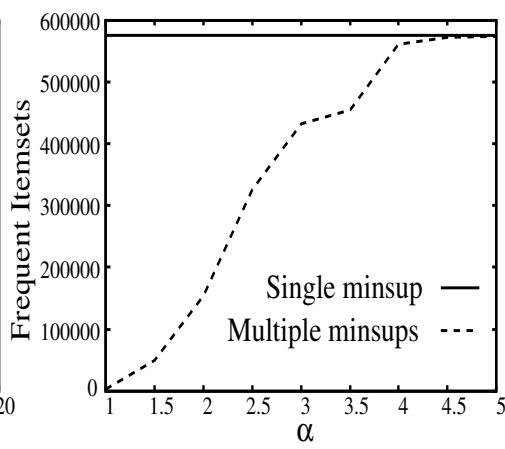

(c) Mushroom dataset

Figure 3: Frequent patterns generated at different $M I S$ values of items.

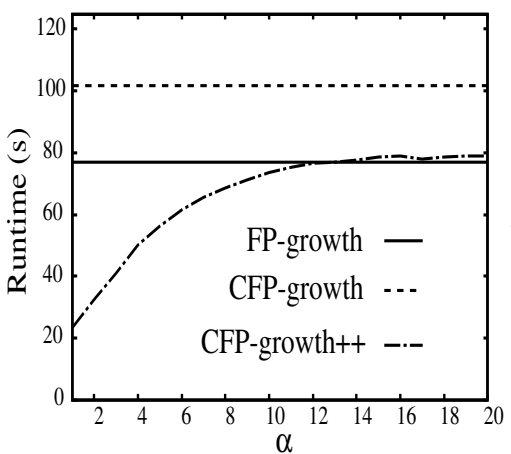

(a) T1014D100K dataset

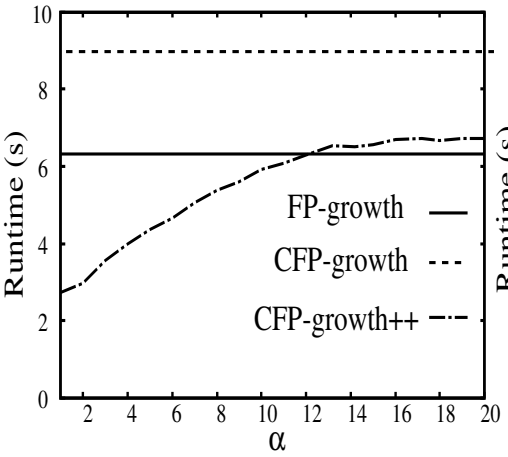

(b) BMS-WebView-1 dataset

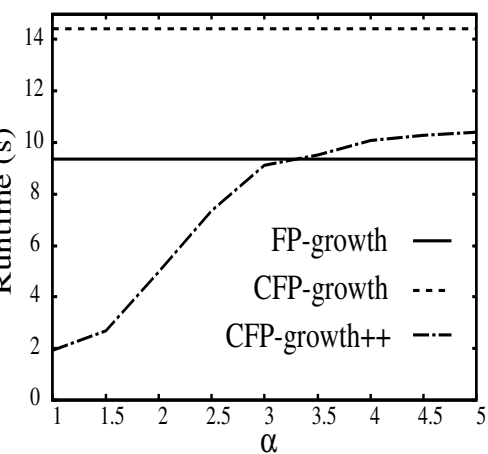

(c) Mushroom dataset

Figure 4: Runtime taken for generating frequent patterns.

on different datasets are shown in the Figures 4(a), 4(b) and 4(c). For CFP-growth and CFP-growth++ algorithms, runtime includes the construction of MIS-tree, construction of compact MIS-tree and mining frequent patterns from compact MIS-tree. For FP-growth algorithm, runtime includes the construction of FP-tree and mining frequent patterns from the FP-tree.

It can be observed that the runtime performance of the FP-growth is significantly better than the CFP-growth for all datasets independent of the $\alpha$ value. It is due to the fact that CFP-growth carries out exhaustive search as "multiple minsups framework" does not satisfy downward closure property, whereas FP-growth exploits downward closure property to reduce the search space. It can be observed that at all $\alpha$ values, the CFP-growth ++ performance is significantly better than the CFP-growth. The performance gap is much higher at lower $\alpha$ values. It is due to the effect of pruning techniques employed by $\mathrm{CFP}$-growth ++ to reduce the search space.

It can also be observed that at higher values of $\alpha$, the runtime consumed by the CFP-growth++ is more than the FP-growth. The difference is more clear in Figures 4(b) and $4(\mathrm{c})$. The reason is as follows. The CFP-growth++ algorithm has to consider $M I S$ value of the suffix item to specify conditional minsup value for its conditional pattern base. Whereas, the FP-growth algorithm simply specifies a constant minsup for a conditional pattern base independent of the suffix item.

In [10], it was mentioned that in many real-world applications, the frequent patterns generated when $\alpha=4$ were interesting to the users. It can be observed that, at $\alpha=4$, the proposed CFP-growth++ improves the runtime performance significantly over CFP-growth.

\subsection{Experiment 2}

In this experiment, we evaluate the scalability performance of CFP-growth and CFP-growth++ algorithms on execution time by varying the number of transactions in a database. We use real-world kosarak dataset for the scalability experiment, since it is a huge sparse dataset. We divided the dataset into five portions of 0.2 million transactions in each part and investigated runtime taken by CFP-growth and CFP-growth ++ algorithms after accumulating each portion with previous parts. For each experiment, we have fixed $\beta=0.25 \%$ and $L S=1 \%$. The experimental result is shown in Figure 5. It can be observed from the graph that as the database size increases, the runtime of both CFP-growth and $\mathrm{CFP}$-growth ++ algorithms increases. However, it can be noted that $\mathrm{CFP}$-growth ++ is more scalable than the CFP-growth algorithm. Overall, CFP-growth ++ is about an order of magnitude faster than the CFP-growth in large databases, and this gap grows wider with the increase in dataset size.

\section{CONCLUSIONS}

To mine frequent patterns containing both frequent and rare items, "multiple minsups framework" was proposed in the literature. By considering "multiple minsups framework," CFP-growth algorithm has been proposed to extract 


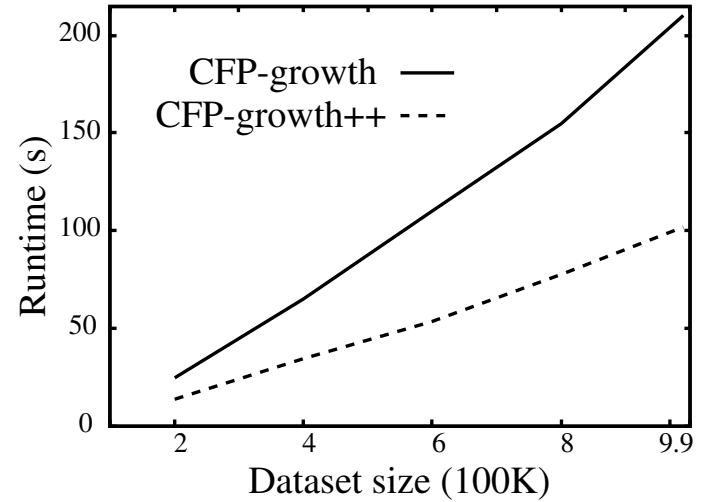

Figure 5: Scalability with number of transactions.

frequent patterns. In this paper, we have proposed an improved CFP-growth algorithm, called CFP-growth ++ , by introducing the following pruning techniques: least minimum support, conditional minsup, conditional closure property and infrequent leaf node pruning. By conducting experiments on both synthetic and real-world datasets, we have shown that the proposed algorithm improves the performance significantly over the exciting approaches.

As a part of future work, we are planning to conduct extensive experiments by considering different types of datasets. It is interesting to investigate how the proposed pruning techniques can be extended to improve the performance of generalized multiple-level frequent patterns.

\section{REFERENCES}

[1] R. Agrawal, T. Imieliński, and A. Swami. Mining association rules between sets of items in large databases. SIGMOD Rec., 22:207-216, June 1993.

[2] R. Agrawal and R. Srikant. Fast algorithms for mining association rules in large databases. In Proceedings of the 20th International Conference on Very Large Data Bases, VLDB '94, pages 487-499, 1994.

[3] M. Hahsler. A model-based frequency constraint for mining associations from transaction data. Data Min. Knowl. Discov., 13(2):137-166, 2006.

[4] J. Han, H. Cheng, D. Xin, and X. Yan. Frequent pattern mining: current status and future directions. Data Min. Knowl. Discov., 15(1):55-86, 2007.

[5] J. Han, J. Pei, Y. Yin, and R. Mao. Mining frequent patterns without candidate generation: A frequent-pattern tree approach. Data Min. Knowl. Discov., 8(1):53-87, 2004.

[6] Y.-H. Hu and Y.-L. Chen. Mining association rules with multiple minimum supports: a new mining algorithm and a support tuning mechanism. Decis. Support Syst., 42(1):1-24, 2006.

[7] R. U. Kiran and P. K. Reddy. An improved frequent pattern-growth approach to discover rare association rules. In Proceedings of the International Conference on Knowledge Discovery and Information Retrieval, pages $43-52,2009$.

[8] R. U. Kiran and P. K. Reddy. An improved multiple minimum support based approach to mine rare association rules. In IEEE Symposium on Computational Intelligence and Data Mining, pages 340-347, 2009.
[9] R. U. Kiran and P. K. Reddy. Mining rare association rules in the datasets with widely varying items' frequencies. In DASFAA (1), pages 49-62, 2010.

[10] B. Liu, W. Hsu, and Y. Ma. Mining association rules with multiple minimum supports. In KDD '99: Proceedings of the fifth ACM SIGKDD international conference on Knowledge discovery and data mining, pages 337-341. ACM, 1999.

[11] C. S. K. Selvi and A. Tamilarasi. Mining association rules with dynamic and collective support thresholds. International Journal on Open Problems Computational Mathematics, 2(3):427-438, 2009.

[12] R. Uday Kiran and P. Krishna Reddy. Towards efficient mining of periodic-frequent patterns in transactional databases. In Database and Expert Systems Applications, volume 6262 of Lecture Notes in Computer Science, pages 194-208. Springer, 2010.

[13] G. M. Weiss. Mining with rarity: a unifying framework. SIGKDD Explor. Newsl., 6(1):7-19, 2004.

[14] H. Yun, D. Ha, B. Hwang, and K. H. Ryu. Mining association rules on significant rare data using relative support. J. Syst. Softw., 67:181-191, September 2003.

[15] Z. Zheng, R. Kohavi, and L. Mason. Real world performance of association rule algorithms. In $K D D$ '01: Proceedings of the seventh ACM SIGKDD international conference on Knowledge discovery and data mining, pages 401-406. ACM, 2001.

[16] L. Zhou and S. Yau. Association rule and quantitative association rule mining among infrequent items. In International Workshop on Multimedia Data Mining, 2007. 